\title{
Energy Efficient Duty Cycle Design based on Quantum Immune Clonal Evolutionary Algorithm in Body Area Networks
}

\author{
Jie Zhou \\ Department of Engineering \\ Macquarie University \\ Sydney, Australia \\ jie.zhou8@students.mq.edu.au \\ Ren Ping Liu \\ Commonwealth Scientific and \\ Industrial Research \\ Organisation (CSIRO) \\ ren.liu@csiro.au
}

\author{
School of Electronics Eng. \\ Beijing University of \\ Post and Telecommunications \\ Beijing, China \\ Gengfa Fang \\ Department of Engineering \\ Macquarie University \\ Sydney, Australia \\ Gengfa.Fang@mq.edu.au
}

\author{
Eryk Dutkiewicz \\ Department of Engineering \\ Macquarie University \\ Sydney, Australia \\ eryk.dutkiewicz@mq.edu.au \\ Yuanan Liu \\ School of Electronics Eng. \\ Beijing University of \\ Post and Telecommunications \\ Beijing, China \\ yuliu@bupt.edu.cn
}

\begin{abstract}
Duty cycle design is an important topic in body area networks. As small sensors are equipped with the limited power source, the extension of network lifetime is generally achieved by reducing the network energy consumption, for instance through duty cycle schemes. However, the duty cycle design is a highly complex NP-hard problem and its computational complexity is too high with exhaustive search algorithm for practical implementation. In order to extend the network lifetime, we proposed a novel quantum immune clonal evolutionary algorithm (QICEA) for duty cycle design while maintaining full coverage in the monitoring area. The QICEA is tested, and a performance comparison is made with simulated annealing (SA) and genetic algorithm (GA). Simulation results show that compared to the SA and the GA, the proposed QICEA can extending the lifetime of body area networks and enhancing the energy efficiency effectively.
\end{abstract}

\section{Categories and Subject Descriptors}

G.2.1 [Mathematics of Computing]: Discrete Mathematics - Combinatorics; F.2.2 [Theory of Computation]: Analysis of Algorithms and Problem Ccomplexity-Nonnumerical Algorithms and Problems

\section{General Terms}

Algorithms

\section{Keywords}

Wireless sensor networks, duty cycle design, evolutionary algorithm

\section{INTRODUCTION}

The recent advances in micro-sensor and technology growth in the field of wireless communications has facilitated the deployment of more capable body area networks to monitor and manage patients intelligently. A body area network generally consists of a number of sensor nodes for exact sensing with a wide range of potential disease. Limited by their size, small wireless sensors are generally low-cost, low-power devices with the restricted power source and limited sensing range. Due to the limited power source, Lifetime optimization through duty cycle design has always been an important issue of the body area networks. Careful duty cycle design can be an effective optimization means for achieving the maximal network lifetime with full coverage constraints. However, the computational complexity of duty cycle design problem increases exponentially with the increment of the number and the lifetime of nodes. Thus, the duty cycle design problem can be formulated as a nonlinear combinational optimization problem and its computational complexity is too high with exhaustive search algorithm for practical implementation[1].

Fortunately, the heuristic algorithms provide a perfect solution to the nonlinear combinational optimization problem, which include the particle swarm optimization (PSO), the genetic algorithm (GA) and the simulated annealing (SA). A research effort to the duty cycle design problem based on the greedy algorithm can be found in [2]. Their work focused on improving the scheduling scheme for sensor sets. The greedy algorithm is simple and fast, but it usually yields shorter network lifetime than evolutionary algorithms. GA method was first introduced in [3]. In [3], the improved GA were proposed for optimizing node scheduling in wireless sensor networks. Their algorithms have shown to perform well on a sensor networks with limited number of nodes. But it suffers from a premature convergence and low 
convergence rate when the number of nodes is high. A duty cycle design scheme is proposed in [4] and PSO is used to solve the problem. However, in practice, it gets stuck into local optima and falls into evolutionary stagnation.

Based on the quantum theory, we proposed a quantum immune clonal evolutionary algorithm (QICEA) for duty cycle design in body area networks which can achieve a longer network lifetime while maintaining full coverage in the monitoring area. QICEA combines quantum computation and artificial immune clonal algorithm, where the elements of the antibody population are Q-bits. Simulation results show that this QICEA for duty cycle design problem in body area networks enjoys longer network lifetime compared to the conventional GA and SA method while maintaining full coverage in the monitoring area.

\section{THE SYSTEM MODEL}

This section describes the system model of the duty cycle design problem with respect to the limited sensing range, the restricted node energy, and the full coverage constraint. Cardei have used a similar model to demonstrate that the duty cycle design problem in sensor networks is NP hard[2]. In this work, a network lifetime system based on round units is employed to model the duty cycle design problem.

Consider a body area network system with $M$ targets and $N$ sensor nodes distributed over the monitoring area, the sensing relationship can be shown as

$$
\begin{array}{r}
R=\left[\begin{array}{ccccc}
r_{1,1} & r_{1,2} & \cdots & r_{1, M-1} & r_{1, M} \\
r_{2,1} & r_{2,2} & \cdots & r_{2, M-1} & r_{2, M} \\
\vdots & & & & \vdots \\
r_{N-1,1} & r_{N-1,2} & \cdots & r_{N-1, M-1} & r_{N-1, M} \\
r_{N, 1} & r_{N, 2} & \cdots & r_{N, M-1} & r_{N, M}
\end{array}\right] \\
\left(r_{n, m} \in\{0,1\}\right) \\
\end{array}
$$

where $r_{n, m}$ is the sensing relationship between the $m$-th target and $n$-th sensor , $r_{n, m}=1$ stands for the $m$-th target is within the sensing range of the $n$-th sensor, and $r_{n, m}=0$ otherwise.

Assume that the unit of lifetime is round and the maximum lifetime of each sensor node is $D$ rounds, the duty cycle of the body area network can be represented as

$$
L=\left[\begin{array}{ccccc}
l_{1,1} & l_{1,2} & \cdots & l_{1, N-1} & l_{1, N} \\
l_{2,1} & l_{2,2} & \cdots & l_{2, N-1} & l_{2, N} \\
\vdots & & l_{i, n} & & \vdots \\
l_{N D-1,1} & l_{N D-1,2} & \cdots & l_{N D-1, N-1} & l_{N D-1, N} \\
l_{N D, 1} & l_{N D, 2} & \cdots & l_{N D, N-1} & l_{N D, N}
\end{array}\right]
$$

where $l_{i, n}=1$ represent the $n$-th sensor is in active mode in round $i$, and $l_{i, n}=0$ represent the $n$-th sensor is in sleep mode in round $i$. The monitoring cycle can be formulated as the form of matrix multiplication, which can be represented as

$$
L R=\left[\begin{array}{ccc}
\sum_{n=1}^{N} l_{1, n} r_{n, 1} & \ldots & \sum_{n=1}^{N} l_{1, n} r_{n, M} \\
\vdots & \sum_{n=1}^{N} l_{i, n} r_{n, m} & \vdots \\
\sum_{n=1}^{N} l_{N D, n} r_{n, 1} & \ldots & \sum_{n=1}^{N} l_{N D, n} r_{n, M}
\end{array}\right]
$$

where $\sum_{n=1}^{N} l_{i, n} r_{n, m}>0$ represent the $m$-th target is monitored by at least one sensor in round $i$, and $\sum_{n=1}^{N} l_{i, n} r_{n, m}=0$ otherwise. In order to maintain full coverage in the monitoring area in the $i$-th round, all elements in $i$-th row must be positive. Thus, from top to bottom, if all elements from row 1 to row $i$ is positive, the network lifetime is longer than $i$ round. So the system model of the duty cycle design problem can be represented as

Objective:

$$
f(L)=\text { row_zero }(L R)-1
$$

Subject to:

$$
\sum_{i=1}^{N D} l_{i, n} \leq D, n=1 \ldots N
$$

where row_zero is the row number for the first zero elemen$\mathrm{t}$ that from top to bottom in matrix $L R$. The constraint $\sum_{i=1}^{N D} l_{i, n} \leq D, n=1 \ldots N$ represent that the maximum lifetime of each sensor node is $D$ rounds.

From the system model, we can see that the number of possible duty cycle combination increases exponentially with the sensor number and the maximum lifetime of nodes. So QICEA is proposed to solve this problem.

\section{ENERGY EFFICIENT DUTY CYCLE DE- SIGN BASED ON QICEA}

Based on the quantum and the clonal selection theory, we introduced a QICEA to enhance the performance of traditional evolutionary algorithms. Compared with the traditional iterative optimization methods, QICEA with quantum bit representation can explore the search space with a linear superposition of states. The key steps of QICEA include antibody encoding, initial antibody generation, affinity calculation, antibody selection and clonal mutation operation, etc.

\subsection{Antibody Encoding}

In QICEA, a Q-bit is a kind of probabilistic representation. It can be in the basis state $|0\rangle$, in the basis state $|1\rangle$, or in any superposition of the two states. As the quantum bit can represent a linear superposition of all possible solutions, its population diversity is higher than other representation. It can be defined as

$$
|\psi\rangle=\alpha|0\rangle+\beta|1\rangle
$$

where $\alpha$ and $\beta$ denote the probability amplitudes of the $|0\rangle$ state and the $|1\rangle$ state, which satisfying

$$
|\alpha|^{2}+|\beta|^{2}=1
$$


$|\alpha|^{2}$ and $|\beta|^{2}$ are the probability that specify the probability amplitudes of $|0\rangle$ state and the $|1\rangle$ state.

A quantum antibody, which is a form of the probabilities of states, is a Sine and Cosine representation of all binary solution states and is more straightforward than traditional Q-bits representation. A quantum antibody with $N$ sensors and maximum lifetime of $N D$ rounds can be represented as

$$
Y=\left[\begin{array}{c|c|c|c}
\cos \left(\theta_{1,1}\right) & \cos \left(\theta_{1,2}\right) & \cdots & \cos \left(\theta_{N D, N}\right) \\
\sin \left(\theta_{1,1}\right) & \sin \left(\theta_{1,2}\right) & \ldots & \sin \left(\theta_{N D, N}\right)
\end{array} \mid\right]
$$

where the $\theta$ give the probabilities that the quantum bit exists in states $|0\rangle$ and $|1\rangle$ respectively, which must satisfy

$$
\left\{\begin{array}{l}
\cos ^{2}(\theta)=|\alpha|^{2} \\
\sin ^{2}(\theta)=|\beta|^{2}
\end{array}\right.
$$

\subsection{Initial Antibody Generation}

In QICEA, all the angles of quantum antibody are initialized with the Chebyshev map, which can be represented as

$$
\theta_{j}=2 \pi \times\left|\chi_{j}\right|
$$

where $\chi_{j} \in(-1,1)$ is the Chebyshev chaotic random number, which can be generated as

$$
\chi_{j+1}=\cos \left(\phi \arccos \chi_{j}\right)
$$

where $\phi \geq 2$ is a constant parameter. It means that al1 possible angles appear with a random probability at the beginning.

\subsection{Affinity Calculation}

For the duty cycle design problem, the quantum antibody should be converted to a binary solution for evaluation.

$$
p_{i, n}= \begin{cases}0 & \text { random }[0,1] \leq \cos ^{2}\left(\theta_{i, n}\right) \\ 1 & \text { random }[0,1]>\cos ^{2}\left(\theta_{i, n}\right)\end{cases}
$$

The binary result is calculated for the affinity with formula (4). When the binary solution do not satisfy the constraint (5), we need to reduce the number of working rounds via change some 1 to 0 for the corresponding node in binary solution.

\subsection{Antibody Selection and Clonal Mutation Operation}

Unlike the general QEA, a clonal selection is applied in QICEA to determine the antibody with the highest affinity. We assume there are $S$ antibodies in the population. First all antibodies are ranked in decreasing order of affinity. Then the first $r$ antibodies with higher affinity are selected for cloning. The number of clone copies can be represented as

$$
Z_{k}=\left\lceil\frac{\rho S}{k}\right\rceil
$$

where $Z_{k}$ is the number of copies that cloned by the $k$-th antibody, $\lceil$ is ceil rounding operation and $\rho$ is a multiplying factor that is chosen empirically. In order to enlarge the population size, the total number of cloned antibody Num must satisfy

$$
N u m=\sum_{q=1}^{r} Z_{k} \geq S
$$

Then the clonal mutation is performed on the cloned antibody population with a quantum gate. In QICEA, the state of Num quantum antibodies can be randomly converted by the a rotation gate, which can be represented as

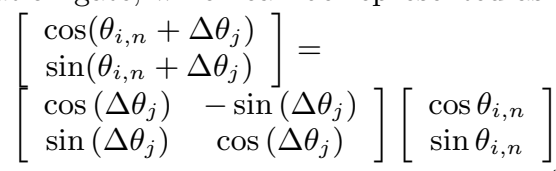

where $\Delta \theta_{j}$ is a random rotation angle for the $(i, n)$ angle in mutation operation. In order to ensure randomness of $\Delta \theta_{j}$, we use the Logistic map to generate $\Delta \theta_{j}$, which can be represented as

$$
\Delta \theta_{j}=\lambda\left(2 x_{j+1}-1\right)
$$

where $\lambda$ is a constant parameter that control the rotation angle, $x_{j+1}$ is generated by the Logistic map, which can be represented as

$$
x_{j+1}=\mu x_{j}\left(1-x_{j}\right), \quad j=0,1,2, \cdots, N D \times N
$$

where $\mu=4$ is a constant parameter of the Logistic map. We use a quantum NOT gate to update the quantum antibody with a small probability, which can be represented as

$$
\left[\begin{array}{ll}
0 & 1 \\
1 & 0
\end{array}\right]\left[\begin{array}{c}
\cos \theta_{i} \\
\sin \theta_{i}
\end{array}\right]=\left[\begin{array}{c}
\sin \theta_{i} \\
\cos \theta_{i}
\end{array}\right]
$$

We can see that probability amplitude of $|0\rangle$ state and $|1\rangle$ state exchanged. After the clonal mutation operation, the QICEA select $S-r$ antibodies from Num quantum antibodies. The combination of these $S-r$ antibodies and the original $r$ antibodies will form a new population with $S$ antibodies.

\subsection{Basic Steps}

The basic steps of QICEA for energy efficient duty cycle design are described in Algorithm 1:

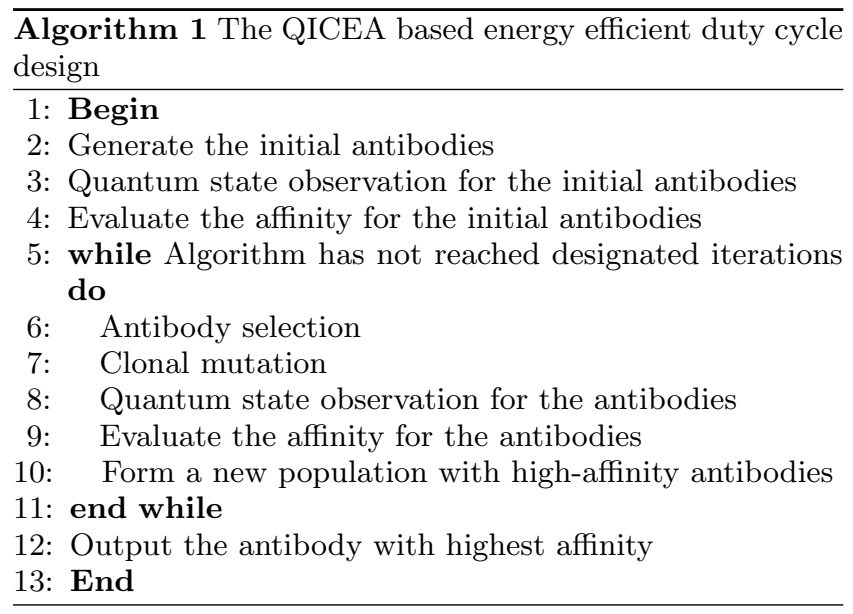

\section{SIMULATION RESULTS AND DISCUS- SION}

In this section, the proposed QICEA is tested with different sensors and targets deployments for the energy efficient duty cycle design problem. The monitoring area is set to $1.5 \times 1.5 \mathrm{~m}$, and the sensor nodes and targets are distributed randomly in it. The sensing radius for each sensor is $0.9 \mathrm{~m}$ and the maximum lifetime of each sensor node is 10 rounds. In QICEA, the number of antibodies is 40. Comparisons are made with GA and SA. In GA, we set the chromosome number is 40 , the crossover probability to 0.9 , the generation gap to 0.9 , the mutation probability to 0.05 . In SA, the 


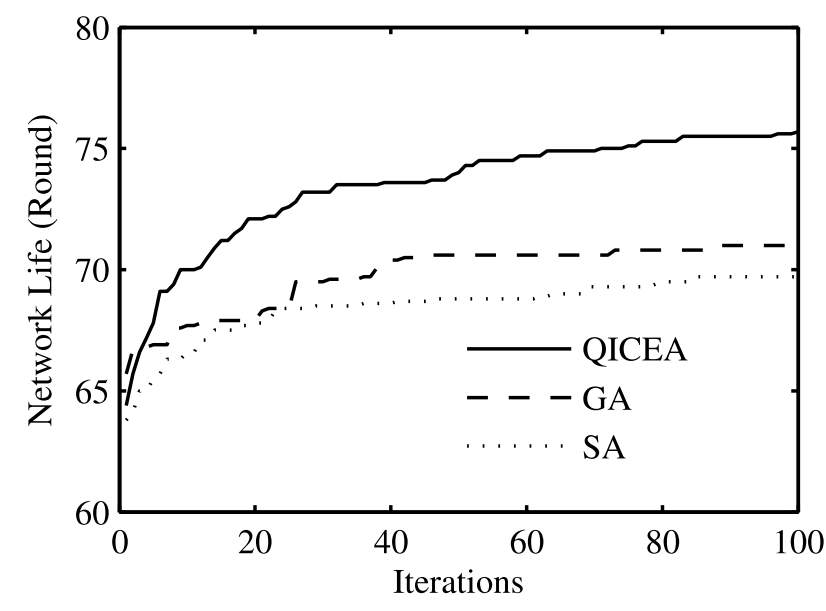

Figure 1: The network lifetime with 10 targets and 55 sensors.

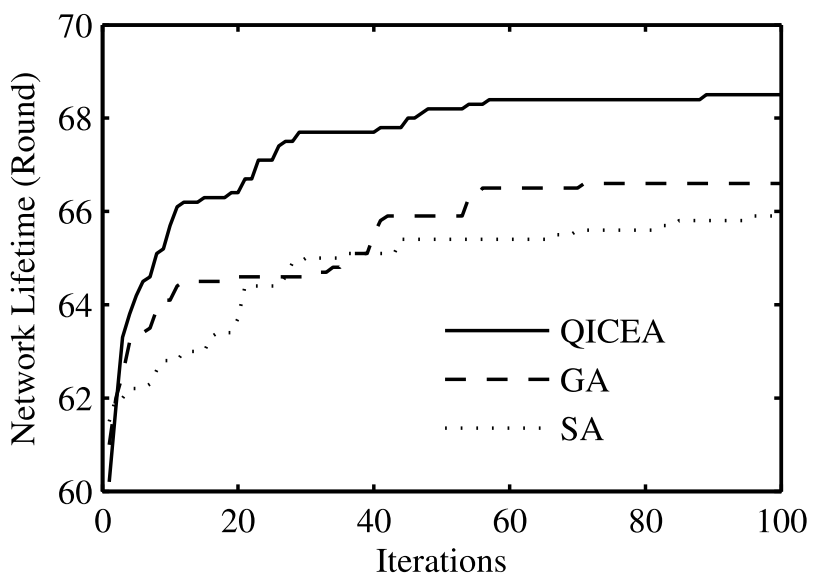

Figure 2: The network lifetime with 20 targets and 55 sensors.

initial temperature is set to 500 degrees, annealing temperature coefficient is set to 0.98 . The maximum iteration of all three algorithms is set to 100 .

Fig.1 to Fig.2 shows the network lifetime of QICEA, GA, and SA on the energy efficient duty cycle design problem with 10 targets, 55 sensors and 20 targets, 55 sensors respectively. From the figures, we can see that our algorithm produces a duty cycle with longer network lifetime in both conditions after 100 iterations. In the beginning, convergence rates of all three algorithms increased. However, SA gets stuck in local optima and is not able to escape it. We can see that the convergence rate is very slow after 20 iterations which mean the algorithm falls into premature convergence. The performance of GA was shown to outperform the SA most of the time in the convergence rate as well as the final network lifetime. It has fast convergence rate at the beginning but then stabilizes after 50 iterations. It can be seen that QICEA finds high-quality duty cycle scheme much faster than SA and GA. It also searches for solutions more efficiently. Simulation results show that in Fig.1 to Fig.2, the network lifetime of the proposed QICEA method is $8.61 \%$ and $3.95 \%$ longer than that of $\mathrm{SA}$ and $6.62 \%$ and $2.85 \%$ longer than that of GA, which means the QICEA has a higher energy efficiency.

\section{CONCLUSIONS}

Duty cycle design is a key problem in the operation of body area networks. In this paper, we proposed a QICEA for duty cycle design while maintaining full coverage in the monitoring area. Simulation results show that compared to the SA and the GA, the proposed QICEA can extending the lifetime of body area networks and enhancing the energy efficiency effectively.

\section{ACKNOWLEDGMENTS}

Jie Zhou is a recipient of a Macquarie University Research Excellence Scholarship and is under a cotutelle PhD arrangement between Macquarie University and Beijing University of Posts and Telecommunications. This work was supported in part by the National Natural Science Foundation of China (No.61170275), the National Science and Technology Major Project (No.2012ZX03001001-002), The research project of Guangdong (No.2011B090400433).

\section{REFERENCES}

[1] Dezun D.,Xiangke L., and Kebin L. Distributed Coverage in Wireless Ad Hoc and Sensor Networks by Topological Graph Approaches. IEEE Transactions on Computerss, 61(10):1417 - 1428, 2012.

[2] Cardei M., Thai M. T., and Li Y. Energy-Efficient Target Coverage in Wireless Sensor Networks. In Proceedings of IEEE INFOCOM 05, pages 1976-1984. IEEE, 2005.

[3] Aslanyan L.,?Aslanyan H., and?Khosravi H. Optimal node scheduling for integrated connected-coveragein wireless sensor networks. In Proc of the Computer Science and Information Technologies (CSIT), pages 1-13. IEEE, 2013.

[4] Navin A. H. and Mirnia M. K. Solving coverage problem in wireless camera-based sensor networks by using a distributed evolutionary algorithm. In Proc of the 2014 5th IEEE International Conference on Software Engineering and Service Science, pages 1076-1079. IEEE, 2014. 